\title{
OsWRKY53 Promotes Abscisic Acid Accumulation to Accelerate Leaf Senescence and Inhibit Seed Germination by Downregulating Abscisic Acid Catabolic Genes in Rice
}

\author{
Wenya Xie, Xinru Li, Shiping Wang and Meng Yuan* \\ National Key Laboratory of Crop Genetic Improvement, National Center of Plant Gene Research, Huazhong Agricultural \\ University, Wuhan, China
}

OPEN ACCESS

Edited by:

Serena Varotto,

University of Padua, Italy

Reviewed by:

Shengjie Dai,

Linyi University, China

Yongfeng Guo,

Tobacco Research Institute, Chinese Academy of Agricultural Sciences

(CAAS), China

*Correspondence:

Meng Yuan

myuan@mail.hzau.edu.cn

Specialty section:

This article was submitted to

Plant Physiology,

a section of the journal

Frontiers in Plant Science

Received: 16 November 2021

Accepted: 29 December 2021

Published: 27 January 2022

Citation:

Xie W, Li X, Wang S and Yuan M

(2022) OsWRKY53 Promotes

Abscisic Acid Accumulation to

Accelerate Leaf Senescence and

Inhibit Seed Germination by

Downregulating Abscisic Acid

Catabolic Genes in Rice.

Front. Plant Sci. 12:816156.

doi: 10.3389/fp/s.2021.816156
Abscisic acid (ABA) largely promotes leaf senescence and inhibits seed germination in plants. Endogenous ABA content is finely tuned by many transcription factors. In this study, we showed that OsWRKY53 is a positive regulator of leaf senescence and a negative regulator of seed germination in rice. OsWRKY53 expression was induced in leaves under aging, dark, and ABA treatment. The OsWRKY53overexpressing (OsWRKY53-oe) plants showed early yellowing leaves, while the OsWRKY53 (oswrky53) knockout mutants maintained green leaves than the wild type under natural, dark-induced, and ABA-induced senescence conditions. Transcriptional analysis revealed that ABA catabolic genes, namely, OsABA80x1 and OsABA8ox2, two key genes participating in ABA catabolism harboring ABA 8'-hydroxylase activity, were markedly downregulated in OsWRKY53-oe leaves. Chromatin immunoprecipitation and protoplast transient assays revealed that OsWRKY53 directly bound to the promoters of OsABA8ox1 and OsABA80x2 to repress their transcription, resulting in elevated endogenous $A B A$ contents that promoted premature leaf senescence in the OsWRKY53-oe plants. It indicates that OsWRKY53 is a positive regulator through regulating $A B A$ accumulation to promote leaf senescence. In addition, accumulated ABA simultaneously inhibited seed germination and post-germination growth in OsWRKY53-oe plants. Taken together, OsWRKY53 suppresses the transcript of ABA catabolic genes to promote ABA accumulation to modulate ABA-induced leaf senescence and ABA-mediated inhibition of seed germination.

Keywords: leaf senescence, seed germination, ABA, OsWRKY53, transcription factor

\section{INTRODUCTION}

Leaf senescence, the final stage of leaf development, is featured with developmentally programmed leaves yellowing due to the loss of green pigment chlorophyll (Lim et al., 2007; Woo et al., 2019). The onset of leaf senescence begins with chlorophyll degradation, while accelerated chlorophyll degradation usually promotes leaf senescence. Leaf senescence is controlled by multiple genetic and 
environmental factors. The major genetic factors are termed senescence-associated genes (SAGs), which exhibit accumulated gene expressions during leaf senescence. So far, many SAGs have been characterized in rice. They are involved in nutrient relocation, macromolecules degradation, transcriptional regulation, and signal transduction (Lee and MasclauxDaubresse, 2021). Rice plants containing these loss-of-function or gain-of-function SAGs usually exhibit delayed or accelerated leaf senescence. Moreover, environmental factors including nutrient status, light quality and length, climate change, water usage, and biotic stress separately or collaboratively modulate leaf senescence (Lee and Masclaux-Daubresse, 2021).

Leaf senescence can be triggered or regulated by a variety of phytohormones, with abscisic acid (ABA), ethylene, jasmonic acid (JA), salicylic acid, brassinosteroid, and strigolactone functioning as inducers, whereas cytokinin, gibberellic acid (GA), and auxin functioning as inhibitors (Lim et al., 2007; Woo et al., 2019). Of these phytohormones, ABA plays a key role in plant to promote leaf senescence. Since ABA concentration is controlled by ABA biosynthetic genes or ABA catabolic genes in plants (Nambara and Marion-Poll, 2005), disruption of ABA catabolic genes or overexpression of ABA biosynthetic genes usually promotes leaf senescence, whereas downregulation of ABA biosynthetic genes or activation of ABA catabolic genes frequently delays leaf senescence (Yang et al., 2014; Gao et al., 2016; Huang et al., 2019). Moreover, ABA can specifically induce the expression of senescence-associated transcription factors to promote leaf senescence, which is termed ABA-induced leaf senescence. $\mathrm{ABA}$ elevates the transcripts of three transcription factors, namely, $A B F 2, A B F 3$, and $A B F 4$, to accelerate chlorophyll degradation, leading to leaf senescence in Arabidopsis (Gao et al., 2016), and induces the expression of transcription factor OsNAP to promote leaf senescence in rice (Liang et al., 2014). Apart from promoting leaf senescence, ABA can inhibit seed germination. Several rice ABA biosynthetic and catabolic genes have been reported to affect seed germination-related phenotype (Sano and Marion-Poll, 2021). For example, ABA biosynthetic gene OsNCED3 involving in ABA accumulation and ABA catabolic gene OsABA8ox1 participating in ABA degradation play essential roles in seed germination or post-germination growth (Mao et al., 2017; Huang et al., 2018; Yu et al., 2021).

Transcription factors including NAC, WRKY, and MYB proteins have been referenced regulating leaf senescence. Transcriptions of these transcription factors are induced or suppressed during the process of leaf senescence (Kim et al., 2016). They positively or negatively modulate leaf senescence by regulating downstream target genes. In rice, OsNAC2 directly suppresses $O s A B A 80 \times 1$ transcript, leading to ABA accumulation that triggers ABA-induced leaf senescence (Mao et al., 2017). OsNAP activates the transcripts of chlorophyll degradation genes, such as RCCR1, SGR, NYC3, and NYC1, resulting in leaf senescence (Liang et al., 2014). Among the 98 OsWRKYs in rice, OsWRKY5, OsWRKY23, OsWRKY42, and OsWRKY93 have been characterized as senescence-related genes, promoting or inhibiting leaf senescence (Jing et al., 2009; Han et al., 2014;
Kim et al., 2019; Li et al., 2021). Other 33 OsWRKYs expressions are induced or repressed during leaf senescence (Li et al., 2021), whether they modulate leaf senescence is largely unknown.

OsWRKY53 has been reported to play critical roles in rice growth and development by regulating plant architecture and seed size (Tian et al., 2017, 2021). It also plays roles in plant responses to biotic stress, including pathogens or herbivore, and abiotic stress, such as wounding (Chujo et al., 2007; Yoo et al., 2014; Hu et al., 2015; Xie et al., 2021). In this study, we found that OsWRKY53 promotes premature leaf senescence and inhibits seed germination by modulating ABA metabolism. By integrating genetic assays and biochemical experiments, we uncovered the molecular mechanism of OsWRKY53-regulated leaf senescence and seed germination with that OsWRKY53 directly bound to the promoters of OsABA8ox1 and $O s A B A 80 \times 2$ to repress their expression, resulting in $A B A$ accumulation, which in turn accelerates leaf senescence and inhibits seed germination.

\section{MATERIALS AND METHODS}

\section{Plant Materials and Treatments}

Plant materials used in this study were OsWRKY53overexpressing (OsWRKY53-oe) plants, OsWRKY53 (oswrky53) mutants, OsWRKY53-GFP plants, and the corresponding wildtype cultivar Zhonghua 11 (ZH11) (Xie et al., 2021). The seeds were sown on seedbeds, and 1 month later, the seedlings were transplanted to the paddy field. For dark-induced senescence experiments, detached leaves from 2-month-old plants were cut into 1-cm leaf disks and incubated in complete darkness with 50- $\mu$ M ABA treatment, or a mock treatment with water only.

\section{Seed Germination and Post-Germination Growth Assays}

The seeds of OsWRKY53-oe, oswrky53, and wild type were harvested at 30 days after heading, threshed and soaked in distilled water, and then spread onto plates covered with wet filter papers. To detect ABA effect on seed germination, seeds of consistent maturity were spread onto plates containing $20 \mathrm{ml}$ of distilled water or $4 \mu \mathrm{M}$ ABA solution. The plates were placed in a chamber under a 14 -h light $/ 10$-h dark cycle at $28^{\circ} \mathrm{C}$. Germination was defined as the emergence of the radical, and the number of germinated seeds was counted every $24 \mathrm{~h}$. Germination rate was calculated as the number of total germinated seeds divided by the number of total seeds spread. Post-germination growth was evaluated by measuring shoot length of seedlings.

\section{Chlorophyll Measurement}

About $50 \mathrm{mg}$ leaves from OsWRKY53-oe, oswrky53, and wild-type plants at the booting stage were sampled for chlorophyll measurement. Rice leaves were incubated in extraction solution (acetone:absolute ethanol:water, 4.5:4.5:1, v/v/v) for $12 \mathrm{~h}$, and the extraction was measured spectrophotometrically at 645 and $663 \mathrm{~nm}$ using a Spark ${ }^{\mathrm{TMM}}$ 
Multimode Microplate Reader (Tecan). The total chlorophyll $(\mathrm{mg} / \mathrm{g})=\left(20.29 A_{645}+8.05 A_{663}\right) \times v / m \times 1,000$, where $v$ is the volume of extraction solution, and $m$ is the mass of leaves.

\section{Measurement of Ion Leakage Rates}

The detached leaves were cut into $1-\mathrm{cm}$ piece and immersed in $8 \mathrm{ml}$ of deionized water in a $10-\mathrm{ml}$ test tube for $24 \mathrm{~h}$ at room temperature with continual shaking at a speed of $100 \mathrm{rpm}$. The initial conductivity (R1) was measured using a Conductivity Meter (DDSJ-308A, Shanghai Leici, China). After that, the test tubes were placed in boiling water for $20 \mathrm{~min}$ and cooled naturally to room temperature, and the conductivity (R2) was documented again. Ion leakage rate was calculated as the ratio of R1 to R2.

\section{Abscisic Acid Measurement}

Three-month-old leaves of OsWRKY53-oe, oswrky53, and wildtype plants were harvested for endogenous ABA measurement. Briefly, three replicates of frozen leaf samples $(\sim 100 \mathrm{mg}$ for each replicate) were pulverized in liquid nitrogen and then homogenized in $80 \%$ methanol. Each sample was extracted twice with extraction solution (methanol:water:glacial acetic acid, $80: 19: 1, \mathrm{v} / \mathrm{v} / \mathrm{v}$ ) via incubation for $12 \mathrm{~h}$ at $4^{\circ} \mathrm{C}$ and centrifuged at $5,000 \mathrm{~g}$ for $30 \mathrm{~min}$. The supernatant was filtered using a nylon filter with $0.22-\mu \mathrm{m}$ pore size (Jinteng, China), and the eluate was measured using an ABA ELISA kit (Shanghai Jianglai Biotechnology, China) according to the instructions of the manufacturer.

\section{Gene Expression Analysis}

For gene expression analysis, total RNA from the leaves of OsWRKY53-oe, oswrky53, and wild-type plants was isolated using Trizol reagent (Invitrogen, United States). cDNAs were synthesized with Superscript II reverse transcriptase (Invitrogen, United States) according to the manufacturer's protocol. Quantitative real-time PCR (RT-qPCR) was performed using LightCycler 480 SYBR Green I Master (Roche, Switzerland) in the ABI 7500 Real-Time PCR System (Applied Biosystems, United States) as described previously (Xie et al., 2021). Genespecific primers were designed using primer analysis software Primer Express version 3.0 (Applied Biosystems, United States). The transcript levels of rice actin gene were used to normalize expression levels for genes (Supplementary Table 1). The transcript levels of examined genes were quantified by a relative quantitation method $\left(2^{-\triangle \Delta C T}\right)$. Each RT-qPCR assay was biologically repeated at least twice with similar results, with each repetition having three technical replicates. The differences were analyzed for statistical significance using two-tailed Student's $t$-test.

\section{Chromatin Immunoprecipitation-qPCR}

Chromatin immunoprecipitation assay was performed as described previously (Xie et al., 2021). Briefly, chromatin was extracted and fragmented via ultrasound to 200-400 bp. Antibodies anti-GFP and IgG as control were incubated with $40 \mu \mathrm{l}$ protein A Dynabeads (Invitrogen, Norway) at $4^{\circ} \mathrm{C}$ for $4 \mathrm{~h}$ after washing the beads, then $100 \mu \mathrm{l}$ fragmented chromatin suspension was added, followed by incubation at $4^{\circ} \mathrm{C}$ for $12 \mathrm{~h}$. After extensive washing and de-crosslinking, the precipitated and input DNA samples were analyzed by qPCR using gene-specific primers (Supplementary Table 1).

\section{Transient Expression Assay in Protoplasts}

The coding sequence of OsWRKY53 was amplified with genespecific primers and cloned into the pU1301-GFP vector as the effector, with the empty pU1301-GFP vector as the control (Xie et al., 2021). The OsABA8ox1 and OsABA80x2 promoters $(1,200 \mathrm{bp}$ upstream of the translational initiation site) from ZH11 were amplified with gene-specific primers (Supplementary Table 1). The amplified PCR products were cloned into the pGreenII-0800 vector as the reporters (Xie et al., 2021). To measure the transcriptional regulation activity, the effector and reporter constructs were co-transformed into rice protoplasts derived from 14-day-old seedlings of rice cultivar ZH11 as described previously (Xie et al., 2021). Rice protoplasts were isolated by digesting rice sheath strips in digestion solution (i.e., $10 \mathrm{mM}$ MES, $\mathrm{pH}$ 5.7, $1 \mathrm{mM} \mathrm{CaCl}$, $0.6 \mathrm{M}$ mannitol, $0.03 \% \beta$-mercaptoethanol, $0.1 \% \mathrm{BSA}, 0.75 \%$ macerozume R10, and $0.3 \%$ cellulase RS) for $4 \mathrm{~h}$. For transformation, $3 \mu \mathrm{g}$ of each plasmid was added together and gently mixed with 100 $\mu \mathrm{l}$ of protoplasts and $110 \mu \mathrm{l}$ of $\mathrm{PEG}-\mathrm{CaCl}_{2}$ solution. The transfected protoplasts were cultured for $12 \mathrm{~h}$ at $25^{\circ} \mathrm{C}$ in the dark and collected by centrifugation at $100 \mathrm{~g}$ for $8 \mathrm{~min}$ and immediately utilized in the luciferase assay. Luciferase activities were measured using a Dual Luciferase Reporter Assay System (Promega, United States) according to the instructions of the manufacturer. The relative reporter gene expression levels were expressed as the ratio of firefly luciferase (LUC) to the renilla luciferase (REN).

\section{Electrophoretic Mobility Shift Assay}

The coding sequence of OsWRKY53 was amplified with genespecific primers (Supplementary Table 1) and cloned into the pCold-TF vector (Xie et al., 2021). The recombinant plasmid was introduced to E. coli BL21 (DE3) cells and then the cells were induced with $0.2 \mathrm{mM}$ isopropylthio- $\beta$ galactoside for $12 \mathrm{~h}$ at $16^{\circ} \mathrm{C}$ and collected by centrifugation. Recombinant His-OsWRKY53 protein was purified using $\mathrm{Ni}$ Sepharose 6 fast Flow (GE-Healthcare, United States). A singlestranded DNA oligonucleotide containing the canonical W-box of OsABA80x1 promoter was synthesized and labeled with 5-carboxyfluorescein (FAM) at its $5^{\prime}$-end (Shanghai Sangon, China). To generate double-stranded oligos, an equal amount of the complementary signal-stranded oligos was mixed, heated to $95^{\circ} \mathrm{C}$ for $2 \mathrm{~min}$, and annealed by gradually cooling down to room temperature. To perform EMSA assay, purified HisOsWRKY53 protein was incubated with FAM-labeled doublestranded oligos and increasing amounts of the unlabeled oligonucleotides (10-, 50-, and 100-fold of labeled probes) at room temperature for $20 \mathrm{~min}$. The samples were finally subjected to electrophoresis on $6 \%$ native acrylamide gels in $0.5 \times$ Trisborate-EDTA buffer under a $4^{\circ} \mathrm{C}$ electrical field of $110 \mathrm{~V}$ for 

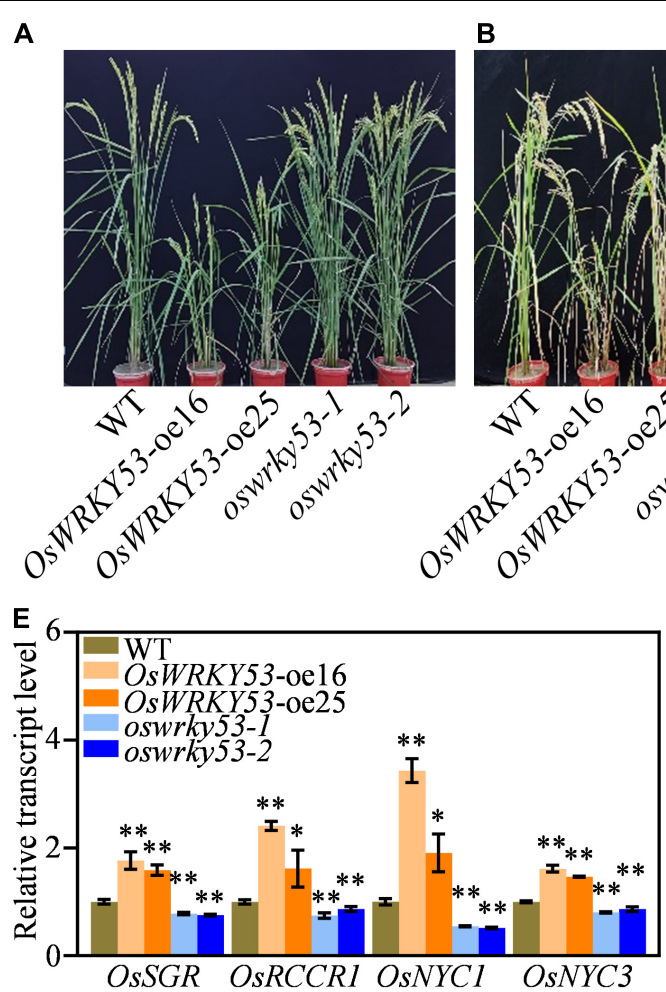

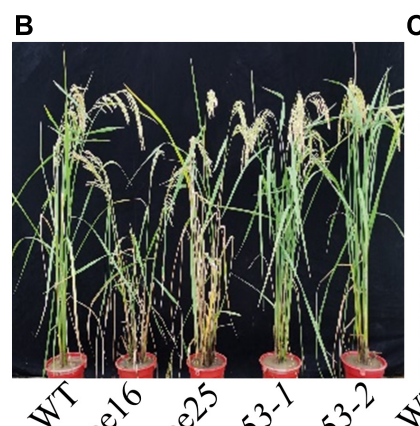

C

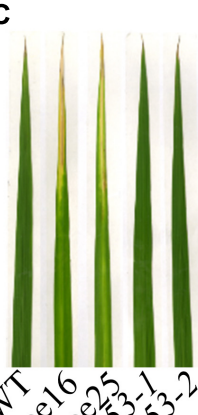

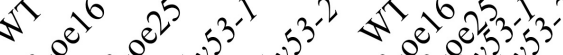

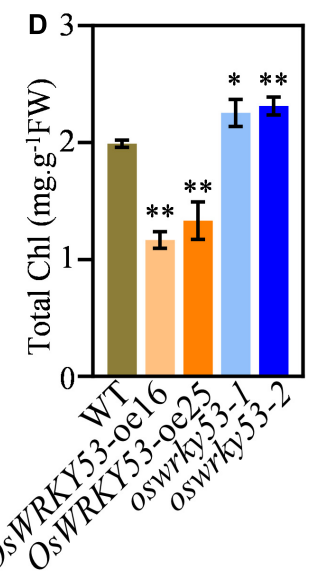

$0^{s} \mathrm{O}^{\mathrm{s}}$
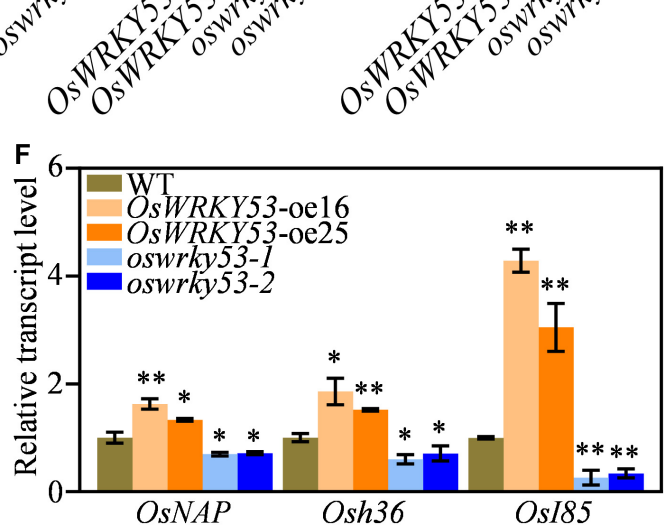

FIGURE 1 | OsWRKY53 promotes natural leaf senescence. (A) Phenotype of OsWRKY53-oe, oswrky53, and wild type (WT) at the heading stage. (B) Phenotype of OsWRKY53-oe, oswrky53, and WT at the grain-filling stage. (C) Senescing flag leaf of OsWRKY53-oe, oswrky53, and WT at the grain-filling stage. (D) Chlorophyll content of senescing flag leaf of OsWRKY53-oe, oswrky53, and WT at the grain-filling stage. (E) Transcript levels of chlorophyll degradation genes in rice leaves at the grain-filling stage. (F) Transcript levels of senescence-associated genes in rice leaves at the grain-filling stage. Data represent mean \pm SD. Asterisks indicate a significant difference determined by two-tailed Student's $t$-test at ${ }^{* *} p<0.01$ or $* p<0.05$.

2 h. Fluorescence signaling was visualized by using FUJIFILM (FLA-5100, Japan).

\section{RESULTS}

\section{OsWRKY53 Promotes Natural Senescence}

We recently reported that OsWRKY53 negatively regulates rice resistance to bacterial pathogen via thickening sclerenchyma cell walls (Xie et al., 2021). When we planted OsWRKY53-oe and oswrky53 knockout plants in the paddy field, there were similar leaf development between OsWRKY53-oe, oswrky53, and wild type at the heading stage (Figure 1A), whereas OsWRKY53-oe plants showed accelerated leaf senescence and oswrky53 mutants exhibited delayed leaf senescence compared with wild type at the grain-filling stage (Figure 1B). OsWRKY53-oe leaves were yellowish, while oswrky53 and wild-type leaves retained green (Figure 1C). As leaf senescence is accompanied with reduced chlorophyll content, this promoted us to determine chlorophyll levels in leaves of these plants. Consistent with the visible accelerated leaf senescence, lower total chlorophyll contents were assessed in OsWRKY53-oe plants than that in oswrky53 mutants and wild type (Figure 1D). We simultaneously assessed the transcript levels of representative chlorophyll degradation genes (e.g., OsRCCR1, OsSGR, OsNYC3, and OsNYC1) and SAGs (e.g., OsNAP, Osh36, and OsI85) in the leaves of these plants (Lee et al., 2001; Jiang et al., 2007; Kusaba et al., 2007; Morita et al., 2009; Tang et al., 2011; Liang et al., 2014; Shin et al., 2020). RT-qPCR assays showed that four chlorophyll degradation gene and three SAGs had higher transcript levels in OsWRKY53-oe plants, but lower levels in oswrky53 mutants relative to them in wild type (Figures 1E,F). In line with OsWRKY53 regulating leaf senescence, OsWRKY53 had the highest transcript levels in leaf and sheath rather than in other tissues (Supplementary Figure 1). Together, these results indicate that OsWRKY53 is positively involved in natural leaf senescence.

\section{OsWRKY53 Promotes Dark-Induced Senescence}

To further verify the role of OsWRKY53 involving in leaf senescence, we assessed its transcriptional patterns in leaves under natural senescence. The transcript levels of OsWRKY53 were detected in rice leaves at different developmental stages, from young leaves (YL), moderately senesced leaves (MSL), to senesced leaves (SL). The different expressions of marker SAGs (e.g., OsDOS and OsI57) and diverse chlorophyll contents in $\mathrm{YL}, \mathrm{MSL}$, and SL revealed the senescing status of these 


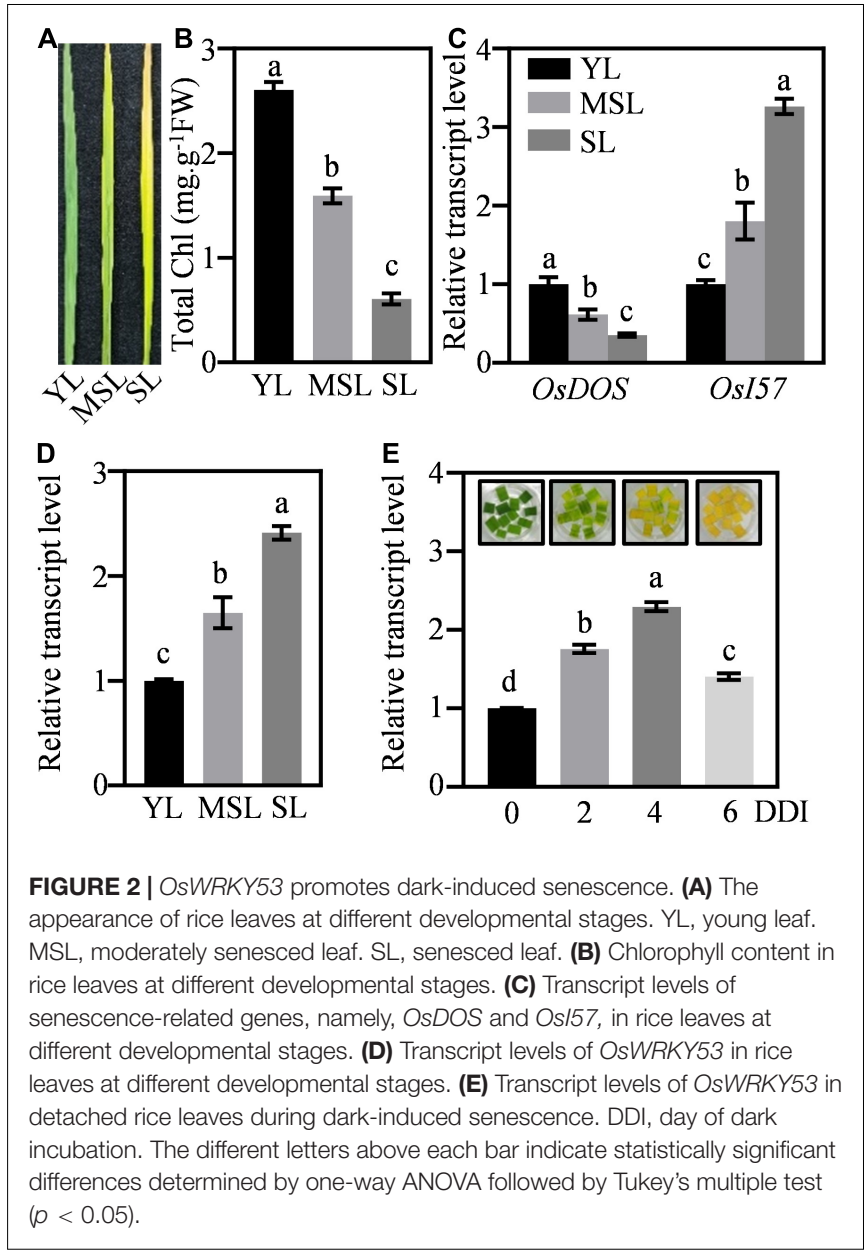

rice leaves (Figures 2A-C). The OsWRKY53 transcripts were increased with leaf development, with the highest level in SL (Figure 2D). Then, we evaluated the response of OsWRKY53 to dark-induced senescence. Along with darkness treatment, rice leaves were yellowish, exhibiting accelerated leaf senescence, and the transcript levels of OsWRKY53 were significantly increased (Figure 2E), suggesting that OsWRKY53 may be a senescencerelated transcription factor.

To explore accelerated leaf senescence caused by overexpression of OsWRKY53 in more detail, we examined the responses of OsWRKY53-oe and oswrky53 mutants under dark treatment. Detached leaf disks from OsWRKY53-oe, oswrky53, and wild-type plants at the booting stage were floated on MES solution and incubated in dark to mimic dark-induced senescence. After 4 days of dark incubation (DDI), the OsWRKY53-oe leaf disks showed yellowish, while oswrky53 leaf disks retained green compared with light green of wild-type leaf disks. After 5 DDI, the accelerated yellowing tendency was greater in leaf disks of OsWRKY53-oe plants than that of wild type or oswrky53 mutants (To explore accelerated leaf senescence caused by overexpression of OsWRKY53 in more detail, we examined the responses of OsWRKY53-oe and oswrky53 mutants under dark treatment. Detached leaf disks from OsWRKY53-oe, oswrky53, and wild-type plants at the booting stage were floated on MES solution and incubated in dark to mimic dark-induced senescence. After 4 days of dark incubation (DDI), the OsWRKY53-oe leaf disks showed yellowish, while oswrky53 leaf disks retained green compared with light green of wild-type leaf disks. After 5 DDI, the accelerated yellowing tendency was greater in leaf disks of OsWRKY53-oe plants than that of wild type or oswrky53 mutants (Figure 3A). Consistent with yellowing leaves, lower chlorophyll contents were observed for OsWRKY53-oe plants, and higher chlorophyll levels were determined for oswrky53 mutants relative to that for wild type (Figure 3B). Accordingly, ion leakage rate, an indicator of membrane disintegration, was higher for OsWRKY53-oe plants, but lower for oswrky53 mutants than wild type under darkness treatment for 4 or 5 days (Figure $3 \mathrm{C}$ ). We also examined the transcript levels of photosystem-related genes, i.e., OsLhcb1 and OsLhcb4, and chlorophyll biosynthetic gene, i.e., OsRbcL, in OsWRKY53-oe, oswrky53, and wild-type plants. The transcript levels of these genes were significantly lower in OsWRKY53-oe plants but higher in oswrky53 mutants than in wild type after 5 DDI (Figure 3D). In parallel, 20-day seedlings of OsWRKY53-oe, oswrky53, and wild type were treated under darkness, with the similar results that OsWRKY53-oe plants exhibited accelerated leaf senescence (Supplementary Figure 2). Taken together, these results indicate that OsWRKY53 promotes dark-induced senescence.

\section{Accumulated Abscisic Acid Contributes to Leaf Senescence for OsWRKY53-oe Plants}

Leaf senescence is regulated by a variety of phytohormones, which act as senescence-promoting phytohormones or senescence-inhibiting phytohormones (Kusaba et al., 2013). To explore the mechanism of OsWRKY53-promoted leaf senescence, we analyzed expressions of phytohormones signaling or biosynthetic genes from the microarray data set of OsWRKY53-oe plants ${ }^{1}$, which has been deposited on public Gene Expression Omnibus (Chujo et al., 2014). A number of phytohormone signaling and biosynthetic genes have increased or decreased the transcript levels in OsWRKY53-oe plants relative to wild type (Supplementary Figure 3). Of these, majority of $\mathrm{ABA}$ signaling and biosynthetic genes have greater expressions in OsWRKY53-oe plants than in wild type, suggesting potential ABA accumulation in OsWRKY53-oe plants. We then measured ABA contents in the leaves of OsWRKY53-oe, oswrky53, and wild-type plants at the grain-filling stage. OsWRKY53-oe plants had 1.5-fold higher ABA level and oswrky53 mutants had 1.3-fold lower ABA content than wild type (Figure 4A).

To explore whether OsWRKY53 is involved in ABA-promoted leaf senescence, we assessed dynamic expression pattern of OsWRKY53 under ABA treatment. Compared with mock control, the transcript levels of OsWRKY53 were increased after exogenous ABA treatment, suggesting that OsWRKY53 is an ABA-activation transcription factor (Supplementary Figure 4).

\footnotetext{
${ }^{1}$ https://www.ncbi.nlm.nih.gov/geo/query/acc.cgi?acc=GSE48500
} 

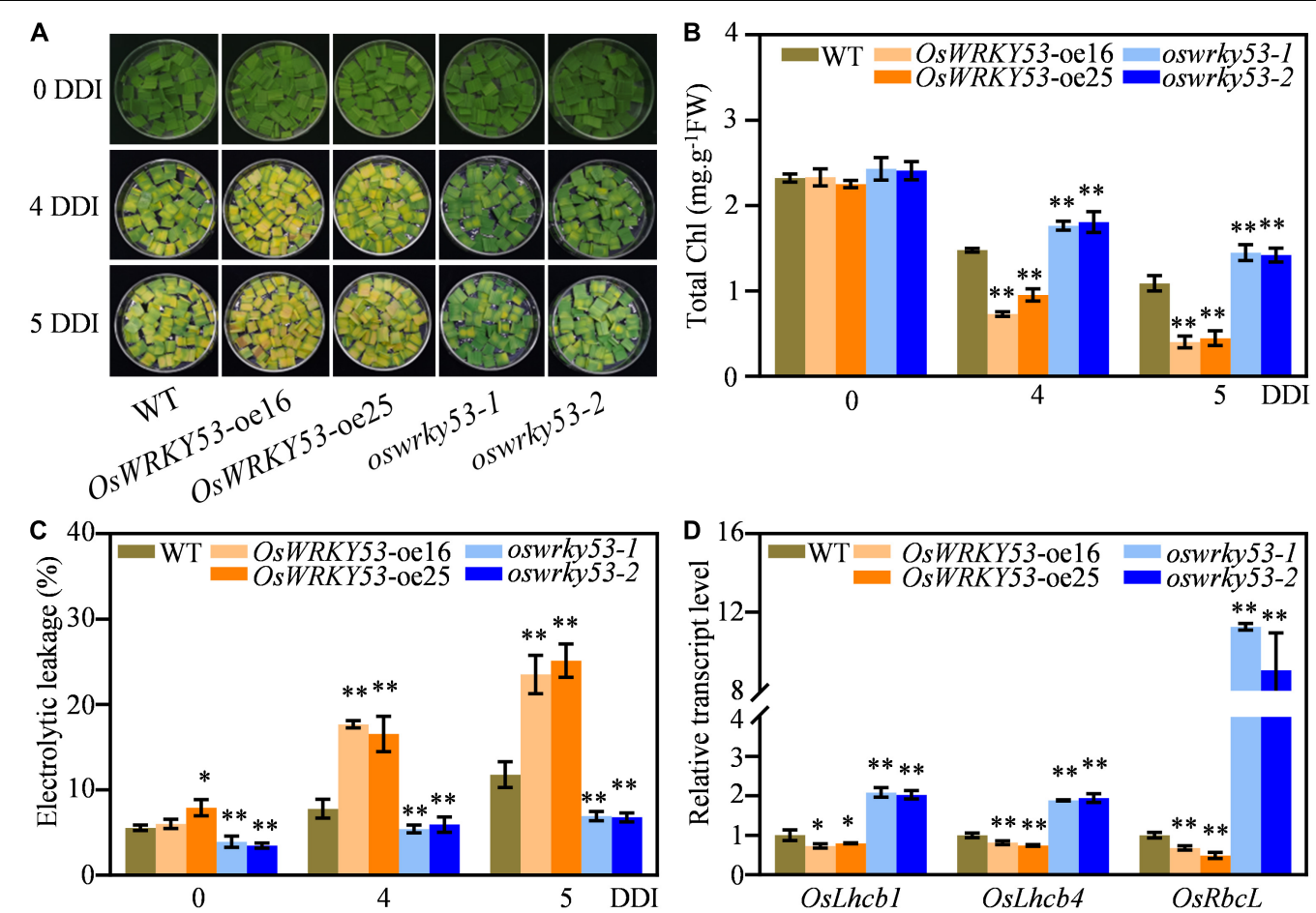

FIGURE 3 | Overexpression of OsWRKY53 promotes dark-induced senescence. (A) Phenotype of detached flag leaf of OsWRKY53-oe, oswrky53, and wild type (WT) at the booting stage after dark treatment. (B) Chlorophyll content of detached flag leaf of OsWRKY53-oe, oswrky53, and WT after dark treatment. (C) lon leakage analysis of detached flag leaf of OsWRKY53-oe, oswrky53, and WT after dark treatment. (D) Relative transcript levels of photosystem-related genes or chlorophyll biosynthetic gene in detached flag leaf of OsWRKY53-oe, oswrky53, and WT after 5 DDI. DDI, day of dark incubation. Data represent mean \pm SD. Asterisks indicate a significant difference between transgenic plants and WT determined by two-tailed Student's $t$-test at ** $p<0.01$ or * $p<0.05$.

We also investigated leaf senescence of OsWRKY53-oe, oswrky53, and wild-type plants under exogenous ABA treatment. We found that exogenous ABA application accelerated leaf senescence for all the leaf disks after darkness treatment, whereas the accelerated senescing tendency was greater for OsWRKY53-oe plants than for oswrky53 mutants and wild type at 3 DDI (Figure 4B). As a result, chlorophyll contents were decreased in all these plants; however, the decreased tendency was significantly greater for OsWRKY53-oe plants (-62\% to 65\%) than for oswrky53 mutants (-9\% to $10 \%)$ and wild type $(-23 \%)$ after exogenous ABA treatment at 3 DDI (Figure 4C). Similarly, ion leakage rates were increased in all these plants, but the increased tendency was greater for OsWRKY53-oe plants (+ 90\% to 103\%) than for oswrky53 mutants (+ $30 \%$ to $31 \%$ ) and wild type (+ $48 \%$ ) after exogenous ABA treatment at 3 DDI (Figure 4D). ABA treatment accelerated leaf senescence for OsWRKY53-oe plants compared with wild type and oswrky53 mutants, indicating that overexpression of OsWRKY53 enhances ABA-promoted leaf senescence. Taken together, these results indicate that OsWRKY53 positively modulates ABA-induced leaf senescence.

\section{OsWRKY53 Regulates Transcripts of Abscisic Acid Catabolic Genes}

The ABA accumulation in OsWRKY53-oe plants promoted us to investigate the transcript levels of ABA metabolism genes. We detected expressions of ABA biosynthetic genes (i.e., OsNCED3, OsNCED4, and OsNCED5) and ABA catabolic genes (i.e., OsABA8ox1, OsABA8ox2, and OsABA8ox3) in the leaves of OsWRKY53-oe, oswrky53, and wild-type plants. These three ABA biosynthetic genes had higher transcript levels in OsWRKY53oe plants, but lower transcript levels in oswrky53 mutants than in wild type (Figure 5A). Contrarily, three ABA catabolic genes had lower transcript levels in OsWRKY53-oe plants, but higher transcript levels in oswrky53 mutants than in wild type (Figure 5B). The enhanced transcript of ABA biosynthetic genes and attenuated expression of ABA catabolic genes supported increased ABA contents in OsWRKY53-oe plants.

OsWRKY53 acts as a transcription repressor by binding to the canonical W-box with TTGACC core sequence at the promoter of its target genes (Xie et al., 2021) OsWRKY53 acts as a transcription repressor by binding to the canonical W-box with TTGACC core sequence at the promoter of its target genes (Xie et al., 2021). Thus, we analyzed whether the above three ABA catabolic genes have $\mathrm{W}$-box at their promoters, and that both OsABA8ox1 and OsABA8ox2 have the canonical W-box at their promoters (Supplementary Figure 5). We carried out EMSA and ChIP assays to evaluate whether OsWRKY53 could directly bind to OsABA8ox1 and OsABA8ox2 promoters. The in vitro EMSA assays showed that purified TF-OsWRKY53 could bind to probe containing "TTGACC" element (Figure 5C). The in vivo ChIP-qPCR 

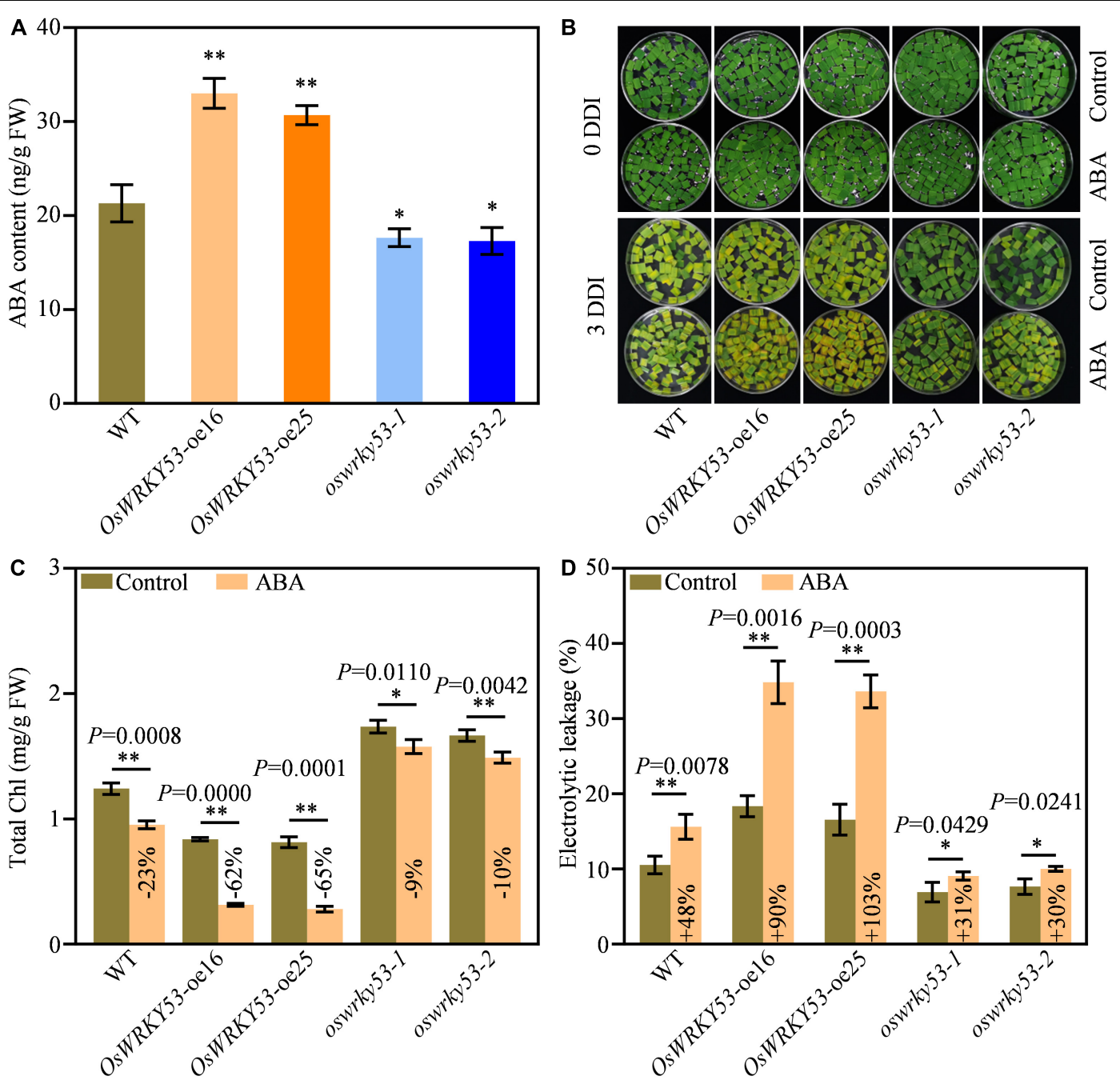

FIGURE 4 | Accumulated ABA promotes leaf senescence in OsWRKY53-oe plants. (A) ABA contents in flag leaf of OsWRKY53-oe, oswrky53, and wild type (WT). Asterisks indicate a significant difference between transgenic plants and WT determined by two-tailed Student's $t$-test at ** $p<0.01$ or * $p<0.05$. (B) Phenotype of detached flag leaf of OsWRKY53-oe, oswrky53, and WT at 3 days of dark incubation (DDI) in the presence of 50 mM ABA and control. (C) Chlorophyll content of detached flag leaf of OsWRKY53-oe, oswrky53, and WT at 3 DDI in the presence of $50 \mu \mathrm{M}$ ABA and control. (D) lon leakage analysis of detached flag leaf of OsWRKY53-oe, oswrky53, and WT at 3 DDI in the presence of $50 \mu \mathrm{M}$ ABA and control. Data represent mean \pm SD. Asterisks indicate a significant difference between exogenous ABA treatment and control determined by two-tailed Student's t-test at ** $p<0.01$ or * $p<0.05$.

assays revealed that enrichment of OsWRKY53 was detected on the $\mathrm{W}$-box-containing region at the promoters of OsABA8ox1 and OsABA8ox2 using anti-GFP antibody in OsWRKY53GFP plants (Xie et al., 2021), but not on other regions without W-box, suggesting direct binding of OsWRKY53 to the promoters of $O s A B A 80 \times 1$ and $O s A B A 80 \times 2$ (Figure 5D). We also conducted transient expression assays to analyze OsWRKY53 action on the expressions of OsABA8ox1 and OsABA8ox2. The LUC reporters driven by $\sim 1.2 \mathrm{~kb}$ promoter of OsABA8ox1 (ProOsABA8ox1:LUC) and OsABA8ox2 (ProOsABA8ox2:LUC) were co-transformed into rice protoplasts with effector constructs ProUbi:GFP and ProUbi:OsWRKY53-GFP. The LUC activities of ProOsABA8ox1:LUC and ProOsABA8ox2:LUC were repressed by OsWRKY53-GFP, but not by ProUbi:GFP, indicating suppressed transcripts of OsABA8ox1 and OsABA8ox2 by OsWRKY53
(Figure 5E). Thus, these results collectively indicate that OsWRKY53 directly binds to OsABA8ox1 and OsABA8ox2 promoters to repress their transcripts.

\section{Overexpression of OsWRKY53 Inhibits Seed Germination and Post-Germination Growth}

ABA not only promotes leaf senescence but also inhibits seed germination and post-germination growth (Gao et al., 2016; Yan and Chen, 2017; Song et al., 2020). To investigate the effect of ABA on germination performance for OsWRKY53 transgenic lines, germination rates were examined using husked full seeds freshly harvested at 30 days after fertilization. Under normal conditions, the oswrky53 mutants and wild-type 
A
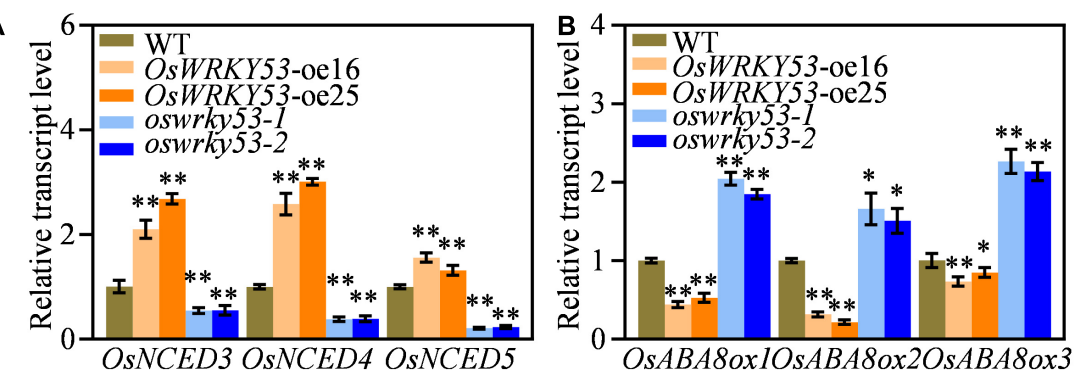

C

$P_{\text {OsABA8oxI }}:$ acaggTTGACCtttgcat

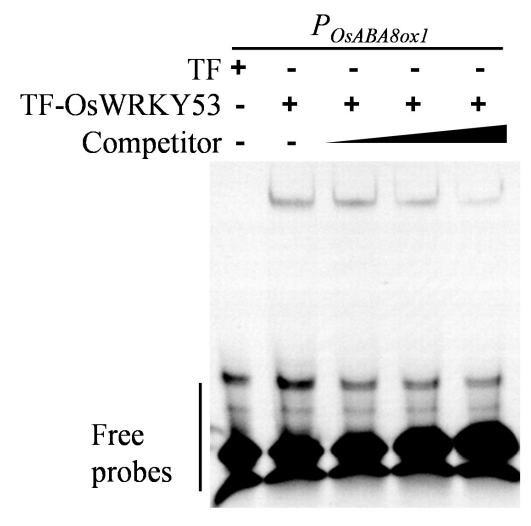

$\mathrm{E}$

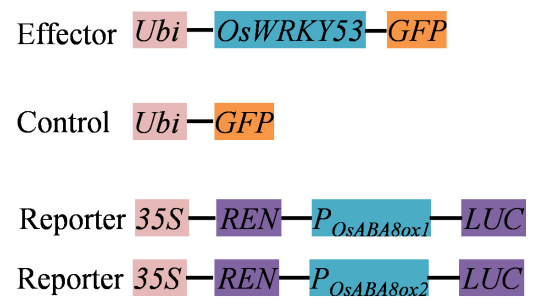

D
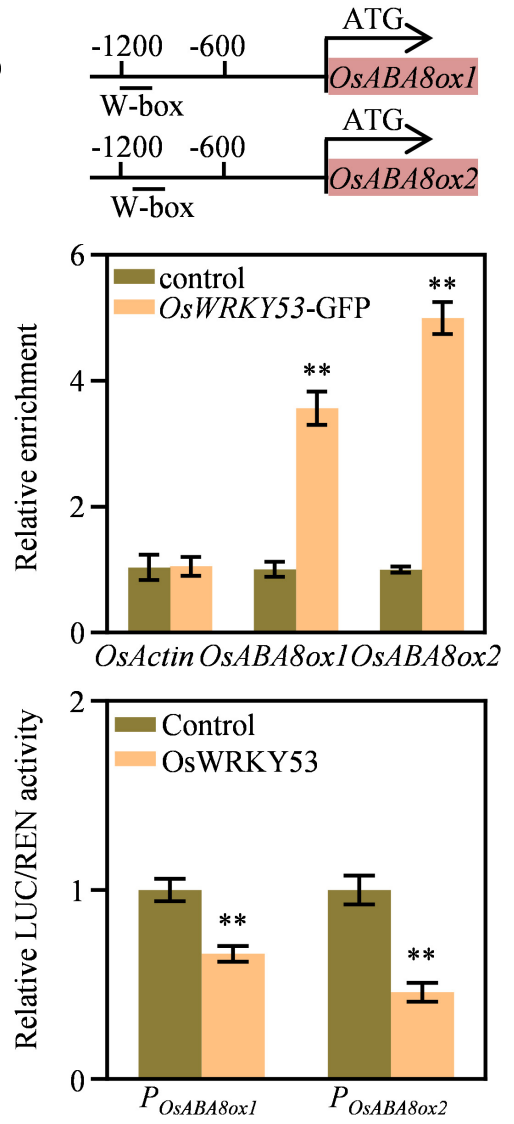

FIGURE 5 | OsWRKY53 binds and downregulates ABA catabolic genes expression. (A) Relative transcript levels of three ABA biosynthetic genes in OsWRKY53-oe, oswrky53 mutants, and wild type (WT). (B) Relative transcript levels of three ABA catabolic genes in OsWRKY53-oe, oswrky53 mutants, and WT. (C) DNA-binding activity assay of OsWRKY53 by EMSA. The red capital letters indicate the canonical W-box. (D) Binding assay of OsWRKY53 to the promoters of OsABA80x1 and OsABA80x2 by ChIP-qPCR in OsWRKY53-GFP plants using the anti-GFP antibody. (E) Activity assay of OsWRKY53 in regulating OsABA80x1 and OsABA80x2 expression. Data represent mean \pm SD. Asterisks indicate a significant difference between transgenic lines and WT determined by two-tailed Student's $t$-test at ${ }^{* *} p<0.01$ or $* p<0.05(\mathbf{A}-\mathbf{C})$, or a significant difference between control and effector determined by two-tailed Student's $t$-test ${ }^{* *} P<0.01$ (D).

seeds rapidly started to germinate after imbibition and the germination rate reached about 18.2 and $11.2 \%$, respectively, at 1 day after imbibition (DAI), while the OsWRKY53-oe plants had only about $4.8 \%$ germination rate at 1 DAI (Figure 6A). At 3 DAI, the oswrky53 mutants and wild-type seeds had germination rate over 82.5 and $77.1 \%$, respectively, which were about threefold higher than that in OsWRKY53oe plants. At 5 DAI, the final germination rate of oswrky53 mutants and wild type were 92.9 and $89.1 \%$, respectively, whereas the germination rate was $30.4 \%$ for OsWRKY53oe plants (Figure 6B), suggesting that overexpression of
OsWRKY53 delays germination and knockout of OsWRKY53 promotes germination.

To investigate the effect of $\mathrm{ABA}$ on post-germination growth for OsWRKY53 transgenic lines, the shoot lengths were examined after seedlings of OsWRKY53-oe, oswrky53, and wild type were subjected to $\mathrm{ABA}$ treatment and non-ABA treatment for 10 days. Under non-ABA treatment, the shoot lengths for OsWRKY53-oe plants, oswrky53 mutants, and wild type were $3.8,7.0$, and $6.2 \mathrm{~cm}$, respectively (Figure $6 \mathrm{C}$ ). The inhibited seedling growth for OsWRKY53-oe lines was consistent with accumulated ABA contents, and the improved seedling growth 

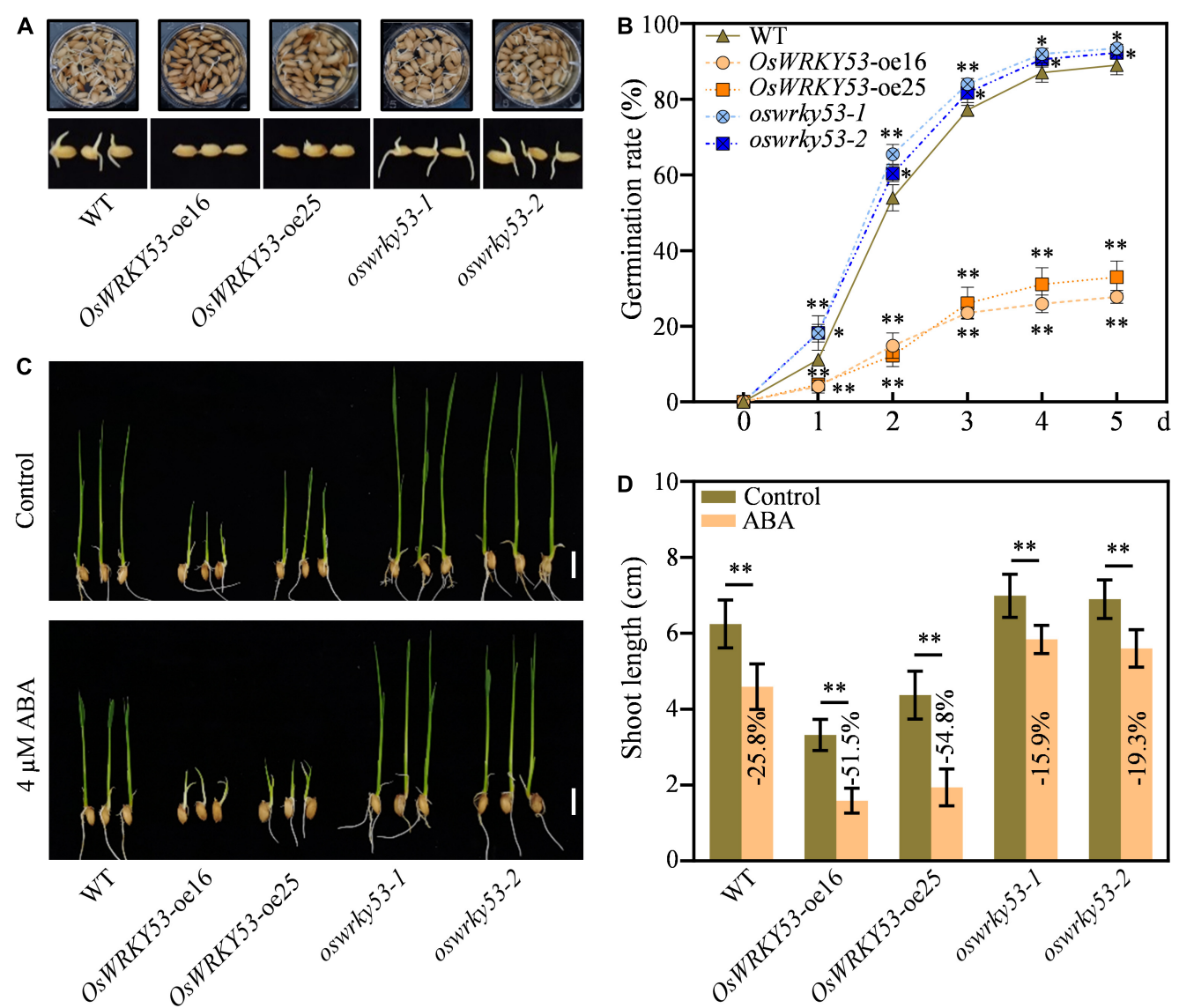

FIGURE 6 | Overexpression of OsWRKY53 inhibits seed germination and post-germination growth. (A) Phenotype of OsWRKY53-oe, oswrky53 mutants, and wild type (WT) at 3 days after imbibition (DAI). (B) Seed germination rate of OsWRKY53-oe, oswrky53 mutants, and WT under normal condition. The germination rate was counted every $24 \mathrm{~h}$ after imbibition. (C) Phenotype of seedlings of OsWRKY53-oe, oswrky53 mutants, and WT at 10 DAl under $4 \mu \mathrm{M}$ ABA treatment with water as a control. Scale bar $=1 \mathrm{~cm}$. (D) Shoot length of OsWRKY53-oe, oswrky53 mutants, and WT at 10 DAl under $4 \mu \mathrm{M}$ ABA treatment with water as a control. Data represent mean \pm SD. Asterisks indicate a significant difference between transgenic lines and WT determined by two-tailed Student's $t$-test at $* * p<0.01$ or ${ }^{*} p<0.05$

for oswrky53 mutants was in line with attenuated ABA levels. Under ABA treatment, the shoot growths were inhibited for all these seedlings, and the shoot lengths for OsWRKY53-oe lines, oswrky 53 mutants, and wild type were $1.8,5.7$, and $4.6 \mathrm{~cm}$, respectively (Figure 6C). The shoot growths were significantly hindered for OsWRKY53-oe plants (-51.5\% to $54.8 \%$ ), but slightly inhibited for oswrky53 mutants ( $-15.9 \%$ to $19.3 \%)$ compared with wild type $(-25.8 \%)$ (Figure 6D). ABA treatment significantly inhibited shoot growth for OsWRKY53-oe plants compared with wild type and oswrky53 mutants, indicating that overexpression of OsWRKY53 increases rice sensitivity to ABA. Collectively, these results indicate that accumulated ABA inhibits seed germination and post-germination growth in OsWRKY53oe plants.

\section{DISCUSSION}

OsWRKY53 has been previously reported to play critical roles not only in rice growth and development but also in responses to biotic and abiotic stress (Chujo et al., 2007; Yoo et al., 2014; Hu et al., 2015; Tian et al., 2017, 2021; Xie et al., 2021). In this study, we found that OsWRKY53 has additional roles in leaf senescence and seed germination.

We found that OsWRKY53 participates in ABA-induced leaf senescence. The transcript of OsWRKY53 was activated by aging, dark, and $\mathrm{ABA}$ treatment (Figures 2D,E). In addition, seven W-boxes and one G-box in the 2,000bp upstream of transcription start codon of OsWRKY53 were identified (Supplementary Figure 6). These ciselements are targeted by WRKY, bZIP, bHLH, and NAC transcription factors, which are involved in the regulation of leaf senescence (Liu et al., 2016). It seems that expression of OsWRKY53 could be regulated by senescence-induced transcription factors or OsWRKY53 is possibly a senescenceinduced transcription factor. Moreover, the progression of leaf senescence was significantly faster in OsWRKY53oe plants than in wild type under natural, dark-induced, and ABA-induced senescence conditions, and the oswrky53 knockout mutants showed delayed leaf senescence. These 
further indicate that OsWRKY53 plays positive roles in leaf senescence.

Senescence-induced transcription factors directly or indirectly regulate expression of SAGs. By analyzing the differentially expressed genes (DEGs) from the microarray data set of OsWRKY53-oe plants, 591 genes were downregulated (Xie et al., 2021). Some of these downregulated DEGs may be the target genes of OsWRKY53 since OsWRKY53 acts as a transcription repressor. We found some senescence-related transcription factors, but not representative SAGs, from these downregulated DEGs, suggesting that OsWRKY53 may directly regulate expression of senescence-related transcription factors to modulate leaf senescence. However, we could not exclude the possibility that OsWRKY53 may indirectly regulate SAGs to promote leaf senescence, because some representative SAGs such as OsNAP, Osh36, and OsI85 had higher expressions in OsWRKY53-oe plants than in wild type (Figure 1F). To investigate whether OsWRKY53 could directly bind to the promoters of senescence-related transcription factors, further biochemical, physiological, and genetic experiments should be carried out.

Apart from SAGs, senescence-related transcription factors can regulate expression of phytohormone metabolic genes to alter phytohormone homeostasis, leading to leaf senescence (Lim et al., 2007; Woo et al., 2019). We found that OsWRKY53 directly bound to the promoters of two ABA catabolic genes, namely, OsABA8ox1 and OsABA8ox2, by recognizing the canonical W-box with EMSA and ChIP-qPCR assays (Figure 5), and suppressed the transcripts of OsABA8ox1 and OsABA8ox2 with transient expression and RT-qPCR assays (Figure 5). OsABA8ox1 is a key gene in ABA catabolism and has ABA 8'-hydroxylase activity in rice. The OsABA8ox1 knockout mutants accumulate ABA, exhibiting premature leaf senescence (Mao et al., 2017). Accordingly, decreased transcripts of these two ABA catabolic genes contributed to ABA accumulation, leading to accelerated leaf senescence for OsWRKY53-oe plants, whereas enhanced expressions of them caused less ABA content, leading to delayed leaf senescence for oswrky53 mutants. It is consistent with that $\mathrm{ABA}$ is generally considered a senescence initiator (Lim et al., 2007; Woo et al., 2019). In addition, there were increased expressions of ABA biosynthetic genes such as OsNCED3, OsNCED4, and OsNCED5 in OsWRKY53-oe plants relative to them in wild type, which may also contribute to $\mathrm{ABA}$ accumulation; however, they are not directly regulated by OsWRKY53. We speculate that these ABA biosynthetic genes may be the targets of senescence-related transcription factors, which are interacting proteins or downstream regulating genes of OsWRKY53. Based on present data, we conclude that OsWRKY53 downregulated transcripts of ABA metabolic genes and OsWRKY53 functions in the promotion of leaf senescence by slowing ABA degradation.

Besides ABA and JA can promote leaf senescence. Several JA biosynthetic genes have higher expressions in the leaves of OsWRKY53-oe plants than in wild type (Supplementary Figure 3). Therefore, we could not exclude the possibility that JA-promoted leaf senescence simultaneously contributes to the accelerated leaf senescence for OsWRKY53-oe plants. Reversely, cytokinin and GA are senescence-inhibiting phytohormones. A number of biosynthetic genes of cytokinin and GA have decreased expressions in OsWRKY53-oe plants than in wild type. Whether OsWRKY53 directly binds and suppresses the expression of these biosynthetic genes, even less contents of cytokinin and GA contribute to the accelerated leaf senescence for OsWRKY53-oe plants should be further elucidated.

Abscisic acid is a negative regulator inhibiting seed germination and post-germination growth. In this study, the OsWRKY53-oe plants accumulating more ABA showed delayed germination after imbibition, lower germination rate, and retarded post-germination growth, whereas the oswrky53 mutants containing less ABA exhibited fast germination, higher germination rate, and greater post-germination growth than wild type (Figure 6). In addition, the OsWRKY53-oe plants were more sensitive to exogenous ABA treatment, indicating that OsWRKY53 is a negative regulator of rice seed germination and post-germination growth.

Integrating previous reports and present results, OsWRKY53 has multiple roles, which is due to either different functions of downstream target genes that it regulates or diverse upstream signals to which it responds. OsWRKY53 plays positive roles in BR signaling, resistance to fungal blast, and a piercing-sucking herbivore and negative roles in resistance to bacterial blight and a chewing herbivore. Thus, the strategy that precisely modulates OsWRKY53 expression to maximize its function for rice genetic improvement is a to be resolved and amazing question.

\section{DATA AVAILABILITY STATEMENT}

The original contributions presented in the study are included in the article/Supplementary Material, further inquiries can be directed to the corresponding author.

\section{AUTHOR CONTRIBUTIONS}

WX and XL performed the experiments. WX analyzed the data and drafted the manuscript. SW and MY supervised the project. MY drafted and revised the manuscript. All authors contributed to the article and approved the submitted version.

\section{FUNDING}

This work was supported by grants from the National Natural Science Foundation of China (31821005, 31871946, and 32172421) and National Science Foundation of Hubei Province (2020CFA058).

\section{SUPPLEMENTARY MATERIAL}

The Supplementary Material for this article can be found online at: https://www.frontiersin.org/articles/10.3389/fpls.2021. 816156/full\#supplementary-material 


\section{REFERENCES}

Chujo, T., Miyamoto, K., Ogawa, S., Masuda, Y., Shimizu, T., Kishi-Kaboshi, M., et al. (2014). Overexpression of phosphomimic mutated OsWRKY53 leads to enhanced blast resistance in rice. PLoS One 9:e98737. doi: 10.1371/journal.pone. 0098737

Chujo, T., Takai, R., Akimoto-Tomiyama, C., Ando, S., Minami, E., Nagamura, Y., et al. (2007). Involvement of the elicitor-induced gene OsWRKY53 in the expression of defense-related genes in rice. Biochim. Biophys. Acta 1769, 497-505. doi: 10.1016/j.bbaexp.2007.04.006

Gao, S., Gao, J., Zhu, X., Song, Y., Li, Z., Ren, G., et al. (2016). ABF2, ABF3, and $\mathrm{ABF} 4$ promote $\mathrm{ABA}$-mediated chlorophyll degradation and leaf senescence by transcriptional activation of chlorophyll catabolic genes and senescenceassociated genes in Arabidopsis. Mol. Plant 9, 1272-1285. doi: 10.1016/j.molp. 2016.06.006

Han, M., Kim, C. Y., Lee, J., Lee, S. K., and Jeon, J. S. (2014). OsWRKY42 represses OsMT1d and induces reactive oxygen species and leaf senescence in rice. Mol. Cells 37, 532-539. doi: 10.14348/molcells.2014.0128

Hu, L., Ye, M., Li, R., Zhang, T., Zhou, G., Wang, Q., et al. (2015). The rice transcription factor WRKY53 suppresses herbivore-induced defenses by acting as a negative feedback modulator of mitogen-activated protein kinase activity. Plant Physiol. 169, 2907-2921. doi: 10.1104/pp.15.01090

Huang, Y., Guo, Y., Liu, Y., Zhang, F., Wang, Z., Wang, H., et al. (2018). 9-cisepoxycarotenoid dioxygenase 3 regulates plant growth and enhances multiabiotic stress tolerance in rice. Front. Plant Sci. 9:162. doi: 10.3389/fpls.2018. 00162

Huang, Y., Jiao, Y., Xie, N., Guo, Y., Zhang, F., Xiang, Z., et al. (2019). OsNCED5, a 9-cis-epoxycarotenoid dioxygenase gene, regulates salt and water stress tolerance and leaf senescence in rice. Plant Sci. 287:110188. doi: 10.1016/j. plantsci.2019.110188

Jiang, H., Li, M., Liang, N., Yan, H., Wei, Y., Xu, X., et al. (2007). Molecular cloning and function analysis of the stay green gene in rice. Plant J. 52, 197-209. doi: 10.1111/j.1365-313X.2007.03221.x

Jing, S., Zhou, X., Song, Y., and Yu, D. (2009). Heterologous expression of OsWRKY23 gene enhances pathogen defense and dark-induced leaf senescence in Arabidopsis. Plant Growth Regul. 58, 181-190. doi: 10.1007/s10725-0099366-z

Kim, H. J., Nam, H. G., and Lim, P. O. (2016). Regulatory network of NAC transcription factors in leaf senescence. Curr. Opin. Plant Biol. 33, 48-56. doi: 10.1016/j.pbi.2016.06.002

Kim, T., Kang, K., Kim, S. H., An, G., and Paek, N. C. (2019). OsWRKY5 promotes rice leaf senescence via senescence-associated NAC and abscisic acid biosynthesis pathway. Int. J. Mol. Sci. 20:4437. doi: 10.3390/ijms20184437

Kusaba, M., Ito, H., Morita, R., Iida, S., Sato, Y., Fujimoto, M., et al. (2007). Rice NON-YELLOW COLORING1 is involved in light-harvesting complex II and grana degradation during leaf senescence. Plant Cell 19, 1362-1375. doi: $10.1105 /$ tpc.106.042911

Kusaba, M., Tanaka, A., and Tanaka, R. (2013). Stay-green plants: what do they tell us about the molecular mechanism of leaf senescence. Photosynth. Res. 117, 221-234. doi: 10.1007/s11120-013-9862-x

Lee, R. H., Wang, C. H., Huang, L. T., and Chen, S. C. (2001). Leaf senescence in rice plants: cloning and characterization of senescence up-regulated genes. $J$. Exp. Bot. 52, 1117-1121. doi: 10.1093/jexbot/52.358.1117

Lee, S., and Masclaux-Daubresse, C. (2021). Current understanding of leaf senescence in rice. Int. J. Mol. Sci. 22:4515. doi: 10.3390/ijms22094515

Li, Y., Liao, S., Mei, P., Pan, Y., Zhang, Y., Zheng, X., et al. (2021). OsWRKY93 dually functions between leaf senescence and in response to biotic stress in rice. Front. Plant. Sci. 12:643011. doi: 10.3389/fpls.2021.643011

Liang, C., Wang, Y., Zhu, Y., Tang, J., Hu, B., Liu, L., et al. (2014). OsNAP connects abscisic acid and leaf senescence by fine-tuning abscisic acid biosynthesis and directly targeting senescence-associated genes in rice. Proc. Natl. Acad. Sci. U.S.A. 111, 10013-10018. doi: 10.1073/pnas.1321568111

Lim, P. O., Kim, H. J., and Nam, H. G. (2007). Leaf senescence. Annu. Rev. Plant Biol. 58, 115-136. doi: 10.1146/annurev.arplant.57.032905.105316

Liu, L., Xu, W., Hu, X., Liu, H., and Lin, Y. (2016). W-box and G-box elements play important roles in early senescence of rice flag leaf. Sci. Rep. 6:20881. doi: $10.1038 /$ srep20881
Mao, C., Lu, S., Lv, B., Zhang, B., Shen, J., He, J., et al. (2017). A rice NAC transcription factor promotes leaf senescence via ABA biosynthesis. Plant Physiol. 174, 1747-1763. doi: 10.1104/pp.17.00542

Morita, R., Sato, Y., Masuda, Y., Nishimura, M., and Kusaba, M. (2009). Defect in non-yellow coloring 3 , an $\alpha / \beta$ hydrolase-fold family protein, causes a staygreen phenotype during leaf senescence in rice. Plant J. 59, 940-952. doi: 10. 1111/j.1365-313X.2009.03919.x

Nambara, E., and Marion-Poll, A. (2005). Abscisic acid biosynthesis and catabolism. Annu. Rev. Plant Biol. 56, 165-185. doi: 10.1146/annurev.arplant. 56.032604.144046

Sano, N., and Marion-Poll, A. (2021). ABA metabolism and homeostasis in seed dormancy and germination. Int. J. Mol. Sci. 22:5069. doi: 10.3390/ijms22105069

Shin, D., Lee, S., Kim, T. H., Lee, J. H., Park, J., Lee, J., et al. (2020). Natural variations at the stay-green gene promoter control lifespan and yield in rice cultivars. Nat. Commun. 11:2819. doi: 10.1038/s41467-020-16573-2

Song, S., Wang, G., Wu, H., Fan, X., Liang, L., Zhao, H., et al. (2020). OsMFT2 is involved in the regulation of $\mathrm{ABA}$ signaling-mediated seed germination through interacting with OsbZIP23/66/72 in rice. Plant J. 103, 532-546. doi: $10.1111 /$ tpj. 14748

Tang, Y., Li, M., Chen, Y., Wu, P., Wu, G., and Jiang, H. (2011). Knockdown of OsPAO and OsRCCR1 cause different plant death phenotypes in rice. J. Plant Physiol. 168, 1952-1959. doi: 10.1016/j.jplph.2011.05.026

Tian, X., He, M., Mei, E., Zhang, B., Tang, J., Xu, M., et al. (2021). WRKY53 integrates classic brassinosteroid signaling and the mitogen-activated protein kinase pathway to regulate rice architecture and seed size. Plant Cell 33, 2753-2775. doi: 10.1093/plcell/koab137

Tian, X., Li, X., Zhou, W., Ren, Y., Wang, Z., Liu, Z., et al. (2017). Transcription factor OsWRKY53 positively regulates brassinosteroid signaling and plant architecture. Plant Physiol. 175, 1337-1349. doi: 10.1104/pp.17.00946

Woo, H. R., Kim, H. J., Lim, P. O., and Nam, H. G. (2019). Leaf senescence: systems and dynamics aspects. Annu. Rev. Plant Biol. 70, 347-376. doi: 10.1146/ annurev-arplant-050718-095859

Xie, W., Ke, Y., Cao, J., Wang, S., and Yuan, M. (2021). Knock out of transcription factor WRKY53 thickens sclerenchyma cell walls, confers bacterial blight resistance. Plant Physiol. 187, 1746-1761. doi: 10.1093/plphys/kia b400

Yan, A., and Chen, Z. (2017). The pivotal role of abscisic acid signaling during transition from seed maturation to germination. Plant Cell Rep. 36, 689-703. doi: 10.1007/s00299-016-2082-z

Yang, J., Worley, E., and Udvardi, M. (2014). A NAP-AAO3 regulatory module promotes chlorophyll degradation via ABA biosynthesis in Arabidopsis leaves. Plant Cell 26, 4862-4874. doi: 10.1105/tpc.114.133769

Yoo, S. J., Kim, S. H., Kim, M. J., Ryu, C. M., Kim, Y. C., Cho, B. H., et al. (2014). Involvement of the OsMKK4-OsMPK1 cascade and its downstream transcription factor OsWRKY53 in the wounding response in rice. Plant Pathol. J. 30, 168-177. doi: 10.5423/PPJ.OA.10.2013.0106

Yu, J., Mao, C., Zhong, Q., Yao, X., Li, P., Liu, C., et al. (2021). OsNAC2 is involved in multiple hormonal pathways to mediate germination of rice seeds and establishment of seedling. Front. Plant Sci. 12:699303. doi: 10.3389/fpls. 2021.699303

Conflict of Interest: The authors declare that the research was conducted in the absence of any commercial or financial relationships that could be construed as a potential conflict of interest.

Publisher's Note: All claims expressed in this article are solely those of the authors and do not necessarily represent those of their affiliated organizations, or those of the publisher, the editors and the reviewers. Any product that may be evaluated in this article, or claim that may be made by its manufacturer, is not guaranteed or endorsed by the publisher.

Copyright (c) $2022 \mathrm{Xie}, \mathrm{Li}$, Wang and Yuan. This is an open-access article distributed under the terms of the Creative Commons Attribution License (CC BY). The use, distribution or reproduction in other forums is permitted, provided the original author(s) and the copyright owner(s) are credited and that the original publication in this journal is cited, in accordance with accepted academic practice. No use, distribution or reproduction is permitted which does not comply with these terms. 